\title{
Erratum: Evidence for Collective Excitations in Superconducting Tin Whiskers*
}

\author{
R. Tidecks \\ I. Physikalisches Institut der Universität Göttingen, Göttingen, West Germany
}

(Received March 10, 1985)

The third footnote on p. 445 should read:

$\$$ For the energy gap without a transport current near $T_{c 0}$ see Ref. 9, pp. 34-35. We consider the reduction of the energy gap by a transport current by setting, for $I \geqslant I_{c}, \Delta(T) \rightarrow$ $(2 / 3)^{1 / 2} \boldsymbol{\Delta}(T)$. This problem has been discussed in Ref. 10 (see the detailed remark in note 45 in the unpublished long version "LV" of that work, copies of which can be obtained from the author). Although the energy gap cannot generally be identified with the superconducting order parameter (see Ref. 9, pp. 119, 261-262), our result should be reasonable near $T_{c 0}$ (see Refs. 11-14).

*This paper appeared in J. Low Temp. Phys. 58, 439 (1985). 